\title{
La lealtad de los clientes de los hostales de la ciudad de Riobamba - Ecuador
}

ReCiBIDO: 13/03/2017 ACEPTADO: 05/06/2017

\section{RESUMEN}

En los últimos años ha habido un creciente incremento de los servicios hoteleros, teniendo una competitividad entre estos, por lo que se hace indispensable la implantación de las estrategias para captar y mantener clientes. Las estrategias aplicadas para mantener clientes llamada lealtad surgen para dar respuesta a esta constante necesidad logrando mejorar los ingresos y reducir los egresos. La lealtad en el sector hotelero viene medida por diferentes factores como la cognitiva, afectiva, conativa y de acción.

Palabras clave: Lealtad, marketing relacional, satisfacción

The Loyalty of THE Customers of THE Hostels OF THE City OF Riobamba- Ecuador

\section{ABSTRACT}

In recent years there has been a growing increase in hotel services, having a competitiveness among them, making it essential to implement strategies to attract and retain customers. The strategies applied to keep customers called loyalty arise to respond to this constant need to improve revenue and reduce outflows. Loyalty in the hotel sector is measured by different factors such as cognitive, affective, conative and action

Keywords: Loyalty, relational marketing, satisfaction.

\author{
Stalin Efrén Arguello Erazo ${ }^{1}$ \\ Margarita Catalina Arguello Erazo ${ }^{2}$ \\ Wilson Manuel Saltos Aguilar ${ }^{3}$
}

\section{INTRODUCCIÓN}

El creciente desarrollo de la industria hotelera debido al auge económico dado por la dolarización, ha dado lugar a que los hoteles y hostales busquen estrategias para captar y mantener clientes. No se han realizado ningún estudio al respecto en la zona central del país.

Al realizar este estudio los propietarios de los establecimientos buscarán que la lealtad de los clientes mejore debido a que conocerán los factores que inciden directa e indirectamente en la lealtad de los huéspedes

Se conocerá los niveles de la lealtad de los clientes gracias al instrumento que permite hacer un test de la lealtad de cada cliente.

La creciente oferta de servicios hoteleros conlleva a la competitividad y a mejorar las estrategias para mantenerse en el mercado. Para Asociation American Marketing (2015) define la Marketing "es la actividad, un conjunto de instituciones y procesos para crear, comunicar, entregar, y el intercambio de ofertas que tienen valor para los consumidores, clientes, socios y la sociedad en general."

Para Chiesa de Negri (2011) señala que el marketing Relacional es un sistema que mantiene y mejora las relaciones con los clientes, además de establecer estrategias para atraer nuevos clientes y las transacciones sean largas y duraderas.

El Marketing relacional se centra más en la adquisición de nuevos clientes buscando una relación duradera que beneficie los intereses del cliente, así como los de la empresa esto se logra mediante la comunicación personalizada, servicio post venta, productos personalizados o de acuerdo a los requerimientos de los clientes y ofertas especiales de los precios de los bienes o servicios ofertados Best (2012)

Para Best (2012) menciona que existen tres niveles de programas de marketing relacional con los clientes: el programa de personalización de las comunicaciones, estrategia de ofertas personalizadas en atención a sus necesidades y comportamien-

1 Docente de la Escuela Superior Politécnica del Chimborazo. Doctor en Gestión de Empresas. E-mail: stalin.arguello@espoch.edu.ec

2 Analista de becas en el Instituto de Fomento al Talento Humano, Magister en Pequeñas y Medianas Empresas mención Finanza.

E-mail: marguello@fomentoacadémico.gob.ec

3 Docente de la Universidad Nacional de Chimborazo, Master en Finanzas. E-mail: wilosaltos@unach.edu.ec 
tos de compra especifico y programas individualizadas de gestión de relaciones.

Sánchez y Segovia (2007) Indica que el Marketing Relacional consiste en establecer, desarrollar y comercializar relaciones a largo, medio y corto plazo con clientes proveedores, distribuidores, entidades laterales (competidores, organizaciones no lucrativas y gobierno) y ámbito interno (empleados, departamentos funcionales y unidades de negocio), tales que permitan el mayor valor ofertado al consumidor final y, a la vez, la consecución de los objetivos de todas partes mencionadas de la forma más eficiente para cada una de ellas.

A pesar que la definición de lealtad ha sido ampliamente estudiado en la literatura sobre servicios existen ciertos desajustes y desacuerdos en su concepto y medida Gil, Moliner, y Ruiz (2009).

La lealtad se puede definir como una promesa de comportamiento relativo al producto o servicio, que conlleva la probabilidad de compras futuras o la posibilidad de que el cliente cambie a otra marca o proveedor del servicio Bigne y Andrew (2014).

La lealtad es una construcción compleja y tres corrientes de distinguir entre la literatura. La primera corriente prefiere definir la lealtad en términos de comportamiento. Nivel de lealtad a la marca se mide mediante el control de la frecuencia de las compras o de la cantidad de cambio de marca entre los consumidores en una categoría de producto. El segundo enfoque es la perspectiva actitudinal, que hace hincapié en que las emociones o los factores afectivos influyen en la lealtad Izquierdo, Cilian, y Gutierrez (2005). La tercera es la perspectiva combinada, que ha examinado la lealtad a la luz de sus dimensiones de comportamiento y de actitud Dick y Basu (2004) han desarrollado un marco para la lealtad del cliente que combine ambas medidas actitudinales y de comportamiento.

"El grado de fidelidad de los clientes se mide como el porcentaje de clientes fieles, el porcentaje de los ingresos asociados a los clientes leales y el surgimiento de cliente fiel después de la ejecución de las actividades de marketing relacional." Izquierdo, Cilian, y Gutierrez (2015).

En la literatura las definiciones de lealtad destacan los compromisos a largo plazo con profundas relaciones con clientes y el negocio específico Dick y Basu (2004); De hecho, la lealtad manifiesta un contrato psicológico profundo entre los clientes y un proveedor de producto / servicio específico debido a que un contrato psicológico surge cuando una de las partes considera que la promesa de rendimientos futuros se ha hecho
Cuando uno viaja a otra ciudad o país, por lo general tiene diferentes necesidades y demandas para el destino uno viaja por paseo, trabajo, estudio $u$ diversión. Tratando de responder a estas necesidades y demandas es lo que comprende la industria del turismo. La industria turística puede ser dividido en sectores principales: la hospitalidad, atracciones eventos, transporte, viajes organizadores y los intermediarios y de los sectores de la organización de destino. Todos estos sectores pueden ser considerados como gama de empresas y organizaciones involucradas en la entrega de los productos turísticos y la experiencia de viaje. Hostales por supuesto, son una parte del sector de la hostelería y, como tal, son los principales componentes de suministro esenciales del turismo. Middleton, Fyall, y Morgan (2011)

Para López (2011) el marketing relacional es el proceso de identificar, mantener, reforzar y de ser necesario terminar las relaciones con los clientes y otros stakeholders de una manera rentable de tal manera que los objetivos de todas las partes involucradas sean logrados y conseguir la lealtad de los clientes.

Esta metodología del marketing relacional es un sistema basado en herramientas tecnológicas que permite construir la lealtad de los clientes. Se fundamente en la idea de establecer con cada cliente una relación de aprendizaje que se forma más inteligente en cada interacción Droskin (2014).

Estos programas, como por ejemplo las millas aéreas, funcionan como descuentos y, por tanto, se basan en incentivos a corto plazo Bejou y Palmer (2008). Ahora bien, no permiten que se construyan relaciones sólidas, por qué no se concentran en satisfacer mejor las necesidades de transporte o de alojamiento de los clientes.

La lealtad se refleja en el incremento de la probabilidad de repetir los comportamientos de compra y de consumo de los mismos productos o servicios que han sido adquiridos consumidos o usados con anterioridad Ramirez y Duque (2013)

La lealtad son las estrategias que se basan en el marketing relacional para lograr que los clientes sean asiduos usuarios de los servicios que ofertan las organizaciones, de la misma manera se busca mediante el marketing relacional tener una relación duradera y que sea a largo plazo con los nuevos clientes y los antiguos clientes.

La lealtad se puede medir en la manera repetitiva del uso del servicio por parte del cliente, todas las estrategias de marketing encaminadas a la consecución de este objetivo o sea de mantener clien- 
tes y que estos sean leales, así como de mantener clientes a largo tiempo se denomina marketing relacional.

El Objetivo de la presente investigación es establecer los niveles de lealtad de los clientes de los hostales de la ciudad de Riobamba Ecuador.

\section{METODOLOGÍA}

De acuerdo a la naturaleza de estudio, según Hernández, Fernandez y Baptista (2010) en su libro describe que existen estos tipos de investigación es descriptiva y explicativa.

Es descriptiva, porque se hace un diagnóstico de las variables de estudio.

Es explicativa, porque pretende identificar las causas de la lealtad mediante el análisis de datos obtenidos en la encuesta.

El diseño de Investigación es pre-experimental pues se aplicó un pre test para diagnosticar el nivel de lealtad.

El presente estudio tiene como unidad de análisis a los clientes que han usado el servicio de los 17 hostales de tal forma que reúnan las condiciones para evaluar dicho servicio y luego realizar un diagnóstico de su grado de lealtad.

La población de estudio que se consideró fue el promedio de clientes que utilizaron los servicios de los hostales de la ciudad de Riobamba que fueron 10 personas por establecimiento y por día, multiplicado por los 17 hostales de estudio da un subtotal de 170 clientes por día, se multiplicó por 26 días que se realizó las encuestas en el mes de mayo del 2015 se excluyó los días domingos, tenemos una población de estudio total de 4420 clientes por mes que visitaron los hostales de la ciudad de Riobamba Ecuador.

La técnica de muestreo fue por conveniencia ya que existen clientes que no disponen de tiempo para responder el cuestionario.

La técnica de muestreo fue aleatoria. El procedimiento para elegir a los participantes de la muestra de la siguiente manera: se dio una numeración a cada pasajero que solicita el servicio y de acuerdo a los números seleccionados con el SPSS aplicando la técnica aleatoria; se procederá a tomar el test de lealtad a dicho cliente.
Para el tamaño de muestra se utilizó la fórmula de:

$$
n=\frac{Z_{\alpha}{ }^{2} \cdot N \cdot p \cdot q}{i^{2}(N-1)+Z_{\alpha}{ }^{2} \cdot p \cdot q}
$$

$Z_{\infty}=1.77$ al cuadrado (si la seguridad es del $92.3 \%$ )

$p=$ proporción esperada (en este caso 0.5 )

$q=1-p($ en este caso $1-0,5=0.5)$

$\mathrm{i}=\operatorname{error}(5 \%)$

$\mathrm{N}=26(\text { días })^{*} 170$ (clientes promedio) $=4420$

$\mathrm{n}=$ desconocido

$p=0.5$

$q=1-0.5=0.5$

$\mathrm{i}=0.077$

Se obtuvo un tamaño de la muestra de 128 clientes.

Para comprobar la fiabilidad se realizó un pre test a 30 personas para luego aplicar el alfa de Cronbach y se obtuvo el siguiente resultado como se muestra en la Tabla 1

Tabla 1. Análisis de confiabilidad.

\begin{tabular}{cc}
\hline \multicolumn{2}{c}{ Estadísticas de fiabilidad } \\
\hline Alfa de Cronbach & N de elementos \\
\hline .941 & 21 \\
\hline
\end{tabular}

Fuente: Elaboración propia.

\section{RESULTADOS}

Del total de encuestados los datos obtenidos en la encuesta según su género son como lo muestra la Tabla 2 a continuación:

Tabla 2. Genero de los encuestados.

\begin{tabular}{lccc}
\hline & Frecuencia & Porcentaje & $\begin{array}{c}\text { Porcentaje } \\
\text { acumulado }\end{array}$ \\
\hline Masculino & 90 & 70.3 & 70.3 \\
\hline Femenino & 38 & 29.7 & 100.0 \\
\hline Total & 128 & 100.0 & \\
\hline
\end{tabular}

Fuente: Elaboración propia. 
De las encuestas realizadas se obtuvo que el $70.3 \%$ son de género masculino y solo un $29.7 \%$ son de género femenino; de aquí se deduce que los hostales prestan los servicios en su mayoría a pasajeros del sexo masculino ya que la mayoría vienen por motivos de comercio o trabajo.

En la Tabla 3 se muestra la principal procedencia de los clientes en los hostales de la ciudad de Riobamba

Tabla 3. Procedencia de los encuestados.

\begin{tabular}{lccc}
\hline & Frecuencia & Porcentaje & $\begin{array}{c}\text { Porcentaje } \\
\text { acumulado }\end{array}$ \\
\hline Guayaquil & 51 & 39.8 & 39.8 \\
\hline Quito & 38 & 29.7 & 69.5 \\
\hline Cuenca & 13 & 10.2 & 79.7 \\
\hline Otro & 26 & 20.3 & 100.0 \\
\hline Total & 128 & 100.0 & \\
\hline
\end{tabular}

Fuente: Elaboración propia.

Se presenta la distribución de frecuencia por lugar de procedencia de los clientes de los hostales de la Ciudad de Riobamba - Ecuador. En la Tabla 3 se observa que el $39.8 \%$ de los clientes son de la ciudad de Guayaquil, el $29.7 \%$ provienen de la ciudad de Quito y el $10.2 \%$ de los clientes son de procedencia de la ciudad de Cuenca.

En la Tabla 4 se muestra los rangos de edad de los clientes que utilizan los servicios de los hostales de la ciudad de Riobamba como a continuación se detalla.

Tabla 4. Edad de los encuestados.

\begin{tabular}{lcc}
\hline & Frecuencia & Porcentaje \\
\hline$(18-30)$ & 19 & 14.8 \\
\hline$(31-40)$ & 77 & 60.2 \\
\hline$(41-50)$ & 19 & 14.8 \\
\hline Mayor a 50 & 13 & 10.2 \\
\hline Total & 128 & 100.0 \\
\hline
\end{tabular}

Fuente: Elaboración propia.

Se observa que el $60.2 \%$ de los encuestados tienen una edad de 31 a 40 años, mientras que el 10.2\% son mayores de 50 años. También se observa que solo el $14.8 \%$ de los encuestados tienen una edad entre 18 a 30 años. $Y$ mayores a 50 años representan el $10.20 \%$
En la Tabla 5 se muestra los valores de la lealtad cognitiva que se tomarán en cuenta para la tabulación de los datos:

Tabla 5. Valores de la lealtad cognitiva.

\begin{tabular}{lc}
\hline \multicolumn{2}{c}{ Cognitiva } \\
\hline Poco & $(0-7)$ \\
\hline Regular & $(8-14)$ \\
\hline Bastante & $(15-20)$ \\
\hline
\end{tabular}

Fuente. Elaboración propia.

En la Tabla 6 se muestra los valores de la lealtad conativa que se tomarán en cuenta para la tabulación de los datos:

Tabla 6. Valores de la lealtad conativa.

\begin{tabular}{lc}
\hline \multicolumn{2}{c}{ Conativa } \\
\hline Bajo compromiso & $(0-7)$ \\
\hline Medio compromiso & $(8-14)$ \\
\hline Alto compromiso & $(15-20)$ \\
\hline
\end{tabular}

Fuente: Elaboración propia.

En la Tabla 7 se muestra los valores de la lealtad afectiva que se tomarán en cuenta para la tabulación de los datos:

Tabla 7. Valores de la lealtad afectiva.

\begin{tabular}{lc}
\hline \multicolumn{2}{c}{ Afectiva } \\
\hline Bajo & $(0-11)$ \\
\hline Medio & $(12-22)$ \\
\hline Alto & $(22-32)$ \\
\hline
\end{tabular}

Fuente: Elaboración Propia

En la Tabla 8 se muestra los valores de la lealtad Acción que se tomarán en cuenta para la tabulación de los datos:

Tabla 8. Valores de la lealtad acción.

\begin{tabular}{lc}
\hline \multicolumn{2}{c}{ Acción } \\
\hline Mala conducta & $(0-4)$ \\
\hline Conducta favorable & $(5-9)$ \\
\hline Buena conducta & $(9-13)$ \\
\hline
\end{tabular}

Fuente: Elaboración Propia 
En la Tabla 9 se muestra los valores que se clasifican los niveles de la lealtad que se tomarán en cuenta para la tabulación de los datos:

Tabla 9. Valores de la lealtad.

\begin{tabular}{lc}
\hline \multicolumn{2}{c}{ Lealtad } \\
\hline Terrorista & $(0-21)$ \\
\hline Desleal & $(22-42)$ \\
\hline Leal & $(43-63)$ \\
\hline Apostos & $(64-88)$ \\
\hline
\end{tabular}

Fuente: Elaboración propia.

En la Tabla 10 se muestra los valores obtenidos en las encuestas de la dimensión Cognitiva obtenido en las encuestas:

Tabla 10. Test de la lealtad cognitiva.

\begin{tabular}{lcc}
\hline & Frecuencia & Porcentaje \\
\hline Poco & 12 & 9.4 \\
\hline Regular & 69 & 53.9 \\
\hline Bueno & 47 & 36.7 \\
\hline Total & 128 & 100.0 \\
\hline
\end{tabular}

Fuente: Elaboración propia.

En la Tabla 10 se presenta la distribución de frecuencia del test de los niveles de lealtad cognitiva de los clientes de los hostales de la Ciudad de Riobamba - Ecuador. Se observa que el $53.9 \%$ de los clientes tiene un conocimiento regular de los servicios de los hostales; es decir, se acuerda como llegar al hostal, conocen su dirección, conocen las promociones y los servicios, para mejorar la estrategia de comunicación de manera regular, y el $36.7 \%$ de los clientes conocen a detalle y bastante respecto a los servicios y ubicación de los hostales.

En la Tabla 11 se muestra los valores obtenidos en las encuestas de la dimensión Conativa obtenido en las encuestas

Tabla 11. Distribución de frecuencia del test de la lealtad conativa de los clientes de los hostales de la Ciudad de Riobamba - Ecuador.

\begin{tabular}{lcc}
\hline & Frecuencia & Porcentaje \\
\hline Bajo compromiso & 3 & 2.3 \\
\hline Medio compromiso & 59 & 46.1 \\
\hline Alto compromiso & 66 & 51.6 \\
\hline Total & 128 & 100.0 \\
\hline
\end{tabular}

Fuente: Elaboración propia.
En la Tabla 11 se presenta la distribución de frecuencia de los niveles de lealtad conativa de los clientes de los hostales de la Ciudad de Riobamba - Ecuador. Se observa que el $2.3 \%$ de los clientes tiene un compromiso bajo con respecto a los servicios de los hostales, el $76.1 \%$ manifiesta un compromiso medio y el $51.6 \%$ presenta un compromiso alto; es decir, que la mayoría de los clientes recomiendan los hostales, manifiestan que ha sido una experiencia buena en su estadía en el hostal y están dispuestos a aconsejar a sus amigos o compañeros.

En la Tabla 12 se muestra los valores obtenidos en las encuestas de la dimensión Afectiva obtenido en las encuestas

Tabla 12. Distribución de frecuencia del test de la lealtad afectiva de los clientes de los hostales de la Ciudad de Riobamba - Ecuador.

\begin{tabular}{lcc}
\hline & Frecuencia & Porcentaje \\
\hline Baja & 4 & 3.1 \\
\hline Alta & 74 & 57.8 \\
\hline Total & 100,0 & 100.0 \\
\hline
\end{tabular}

Fuente: Elaboración propia.

En la Tabla 12 se presenta la distribución de frecuencia de los niveles de lealtad afectiva de los clientes de los hostales de la Ciudad de Riobamba - Ecuador. Se observa que solo el $3.1 \%$ de los clientes tienen una lealtad afectiva baja con respecto a los servicios de los hostales, el $39.1 \%$ tiene una lealtad afectiva media y el $57.8 \%$ presenta una lealtad afectiva alta; es decir, que la mayoría de los clientes $(57.7 \%)$ están satisfechos con los servicios del hostal, consideran que es su hostal preferido, consideran que es confiable y se siente seguros

En la Tabla 13 se muestra los valores obtenidos en las encuestas de la dimensión Acción obtenido en las encuestas

Tabla 13. Distribución de frecuencia de la lealtad en acción de los clientes de los hostales de la Ciudad de Riobamba - Ecuador.

\begin{tabular}{lcc}
\hline & Frecuencia & Porcentaje \\
\hline Mala conducta & 2 & 1.6 \\
\hline Conducta favorable & 79 & 61.7 \\
\hline Buena conducta & 47 & 36.7 \\
\hline Total & 128 & 100.0 \\
\hline
\end{tabular}

Fuente: Elaboración propia. 
En la Tabla 13 se presenta la distribución de frecuencia de los niveles de la lealtad en acción que tienen los clientes de los hostales de la Ciudad de Riobamba - Ecuador. solo el $1.6 \%$ de los clientes tienen una mala conducta con respecto a los servicios de los hostales, el $61.7 \%$ tiene una conducta favorable y el $36.7 \%$ tiene una buena conducta; es decir, que la mayoría de los clientes $(61.7 \%)$ están volverán a utilizar los servicios del hostal, recomendarán y hablan de las buenas experiencias a sus colegas o familiares.

En la Tabla 14 se muestra los valores obtenidos en las encuestas de los niveles de los hostales obtenido en las encuestas

Tabla 14. Distribución de frecuencia de los niveles de lealtad de los clientes de los hostales de la Ciudad de Riobamba - Ecuador

\begin{tabular}{lcc}
\hline & Frecuencia & Porcentaje \\
\hline Terrorista & 0 & 0 \\
\hline Desleal & 15 & 11.7 \\
\hline Leal & 63 & 49.2 \\
\hline Apóstol & 50 & 39.1 \\
\hline Total & 128 & 100.0 \\
\hline
\end{tabular}

Fuente: Elaboración propia.

En la Tabla 14 se presenta la distribución de frecuencia del test de los niveles de la lealtad que tienen los clientes de los hostales de la Ciudad de Riobamba - Ecuador. Se observa que el $11.7 \%$ de los clientes son desleales con respecto a los servicios de los hostales, el $49.2 \%$ tiene son leales y el $39.1 \%$ son apóstoles; es decir, que solo el $39.1 \%$ de los clientes se encuentran satisfechos, comprometidos, recomiendan y hablan bien de los hostales.

\section{CONCLUSIONES Y RECOMENDACIONES}

Con respecto a la dimensión Cognitiva los clientes de los hostales de la ciudad de Riobamba Ecuador desconocen los atributos y características de los servicios que ofertan los hostales de la ciudad de Riobamba Ecuador.

Con respecto a la dimensión Afectiva los clientes de los hostales de la ciudad de Riobamba Ecuador, no tienen una simpatía hacia los servicios que ofertan los hostales de la ciudad de Riobamba-

Con respecto a la dimensión Conativa los clientes de los hostales de la ciudad de Riobamba Ecuador los clientes no recomiendan a utilizar los servicios que ofertan los hostales de la ciudad de Riobamba Ecuador.

Con respecto a la dimensión Cognitiva los clientes de los hostales de la ciudad de Riobamba Ecuador no tienen un hábito de utilizar los servicios de los hostales de la ciudad de Riobamba Ecuador.

Ante esta situación se recomienda que los propietarios de los hostales de la ciudad de Riobamba deben de implementar estrategias como las de Marketing relacional para poder obtener una lealtad por parte de los clientes de los Hostales de esta forma van a tener muchos beneficios por parte de los propietarios como de los clientes.

\section{REFERENCIAS BIBLIOGRÁFICAS}

[1] Asociation American Marketing. (24 de 09 de 2015). www.ama.org. Obtenido de https:// www.ama.org/Pages/default.aspx

[2] Bejou, D., y Palmer, A. J. (2008). Service Failure and Loyalty: An Exploratory Empirical Study of Airline Customers. Journal of Services Marketing, 12(1), 7-22.

[3] Best, R. (2012). Marketing estratégico. México: Pearson Educación S.A.

[4] Bigne, E., y Andrew, L. (2014). Emociones, satisfacción y lealtad del consumidor en entornos comerciales. Un análisis comparativo entre centro comercial y centro urbano. Distribución y Consumo, 77-87.

[5] Chiesa de Negri, C. (2011). CRM: Las cinco Pirámides del marketing relacional. España: Ediciones Deusto.

[6] Dick, A. S., y Basu, K. (2004). Customer Loyaty: Toward an Integrated Conceptual Framework. Journal of Aademy of Marketing Science, 22(2), 99-113.

[7] Droskin, R. (2014). fundamentos de Marketing. México: Ediciones Granica.

[8] Gil, I., Moliner, B., y Ruiz, M. (2009). La formación de lalealtad y su contribucióna la gestión de destinos turisticos . Cuadernos de Administración , 75-98.

[9] Hernández, R., Fernandez, C., y Baptista, P. (2010). Metodología de la Investigación (5ta ed.). México: McGraw Hill.

[10] Izquierdo, C., Cilian, J., y Gutierrez, S. (2015). The impact of customer relationship marketing on thefirm performance: a Spanish case. Journal of Services Marketing, 19(4), 234-244. 
[11] López, B. (2011). Los pilares del Marketing. Catalunya: Universidad Politécnica de Catalunya.

[12] Middleton, V., Fyall, A., y Morgan, M. (2011). Marketing in travel and turism. Oxford.

[13] Ramírez, J., y Duque, J. (2013). Involucramiento de producto y lealtad de marca para productos de consumi masivo. Estudios Gerenciales ICESI.
[14] Rousseau, D. M. (1989). Psychological and Implied Contracts in Organizations. Employee Responsibilities and Rights Journal, 2(2), 121129.

[15] Sánchez, M., y Segovia, C. (2007). La construcción de relaciones de valor en los canales de comercialización. Mediterráneo Económico. Nuevos Enfoques del marketing y la creación del valor. 
